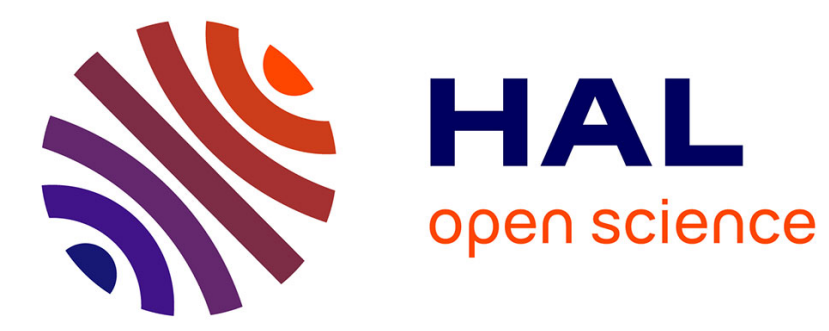

\title{
On regular packings and coverings
}

Jean-Claude Bermond, Johny Bond, Dominique Sotteau

\section{To cite this version:}

Jean-Claude Bermond, Johny Bond, Dominique Sotteau. On regular packings and coverings. Annals of Discrete Mathematics, 1987, 34, pp.81-99. 10.1016/S0304-0208(08)72877-9 . hal-02508731

\section{HAL Id: hal-02508731 \\ https://hal.inria.fr/hal-02508731}

Submitted on 15 Mar 2020

HAL is a multi-disciplinary open access archive for the deposit and dissemination of scientific research documents, whether they are published or not. The documents may come from teaching and research institutions in France or abroad, or from public or private research centers.
L'archive ouverte pluridisciplinaire HAL, est destinée au dépôt et à la diffusion de documents scientifiques de niveau recherche, publiés ou non, émanant des établissements d'enseignement et de recherche français ou étrangers, des laboratoires publics ou privés. 


\title{
On regular packings and coverings
}

\author{
J-C. Bermond, J. Bond, D. Sotteau \\ U.A. 410 CNRS, L.R.I. . \\ bât 490, Université Paris-Sud, 91405 Orsay Cedex, FRANCE
}

TO ALEX ROSA ON HIS YIFTIETH BIRTIDAY

\begin{abstract}
Let us call a regular packing of $K_{n}$ with $K_{k}$ 's a set of edge disjoint subgraphs of $K_{n}$ isomorphic to $K_{k}$ such that the partial graph $G$, called the leave of the packing, generated by the edges not covered is regular. Similarly, a regular covering of $K_{n}$ with $K_{k}$ 's is a set of subgraphs of $K_{n}$ isomorphic to $K_{k}$ which cover all the edges of $K_{n}$ at least once and such that the multigraph $H$, called the excess of the covering, obtained by deleting $K_{m}$ from the union of the $K_{k}$ 's, is regular. Here we study maximum regular packings and minimum regular coverings. More exactly we determine the minimum degrees of the leaves and excesses in case of regular packings and coverings of $K_{n}$ with $K_{3}$ 's and $K_{4}$ 's. We also exhibit minimum regular coverings containing maximum regular packings.
\end{abstract}

\section{Introduction}

A classical problem in design theory concerns the existence of $(n, k, \lambda)$ BIB designs. It is well known that the existence of a $(n, k, 1)$ BIB design is equivalent to the existence of a decomposition of the complete graph $K_{n}$ into $K_{k}$ 's. It is also well known that such a decomposition can exist only if $n(n-1) \equiv 0(\bmod k(k-1))$ and $n-1 \equiv 0(\bmod k-1)$. These conditions have been shown to be sufficient for any $k$ and $n$ large enough (Wilson [18]) and also for $k$ (for a survey on Steiner triple systems, see Doyen and Rosa [7]), $k=4$ and $k=5$ (see Hanani [8]). When $n$ does not meet the necessary conditions one can either try to find the maximum cardinality of a packing of $K_{k}$ 's into $K_{n}$ (that is the maximum number of $K_{k}$ 's included in $K_{n}$ ), or the minimum cardinality of a covering of $K_{n}$ with $K_{k}$ 's (that is the minimum number of edge disjoint $K_{k}$ 's necessary to cover all the edges of $K_{n}$ at least once). Such values have been completely determined for $k=3$ (see Hanani [8]) and for $k=4$ (see Brouwer [5] and Mills [12,13]).

Here we are interested in a slightly different problem that we call regular packing or covering of $K_{n}$ with $K_{k}$ 's. This problem was initially motivated by a problem of interconnection networks, called bus networks of diameter 1, modeled by hypergraphs. In such a network there are $n$ processors connected to buses. Each bus contains the same number $k$ of processors. For every pair of processors there must exist at least one 
bus containing them (diameter 1). These conditions correspond to the classical covering of $K_{n}$ with $K_{k}$ 's. A further requirement (of regularity) is that each processor belongs to the same number of buses, which gives rise to the notion of regular covering (for details see Bermond, Bond and Sacle [3] and[2]).

A regular packing of $K_{n}$ with $K_{k}$ 's is a set of edge disjoint subgraphs of $K_{n}$ isomorphic to $K_{k}$ such that the partial graph $G$, generated by the edges not covered, is regular of degree $d_{G}$. In the literature $G$ is also called the leave of the packing (see $[6])$. In other words we want to find a regular graph $G$ such that $K_{n}-G$ can be decomposed into $K_{k}$ 's. Note that a regular packing of $K_{n}$ with $K_{k}$ 's is just a packing with $K_{k}$ 's such that each vertex of $K_{n}$ belongs to the same number of $K_{k}$ 's. We shall say that a regular packing is maximum if $d_{G}$ is as small as possible.

Similarly, a regular covering of $K_{n}$ with $K_{k}$ 's is a set of subgraphs of $K_{n}$ isomorphic to $K_{k}$ which cover all the edges of $K_{n}$ at least once such that the multigraph $H$, obtained by deleting $K_{m}$ from the union of the $K_{k}$ 's, is regular of degree $d_{H}$. The multigraph $H$ is also called the excess of the covering. In other words we want to find a regular multigraph $H$ of degree $d_{H}$ such that $K_{n}+H$ can be decomposed into $K_{k}$ 's. We shall say that a regular covering is minimum if $d_{H}$ is as small as possible.

Furthermore we are interested in finding, when it is possible, a minimum regular covering of $K_{n}$ with $K_{k}$ 's containing a maximum regular packing. That is equivalent to find whether there exist graphs $G, d_{G}$-regular, and $H, d_{H^{-r e g u l a r}}$, with $d_{G}$ and $d_{H}$ minimum, associated respectively to the packing and covering such that the $\left(d_{G}+d_{H}\right)$ regular graph $G+H$ can itself be decomposed into $K_{k}$ 's. In other words, for that purpose, it is sufficient to find a maximum regular packing such that the $d_{G}$-regular graph $G$ can be covered by $K_{k}$ 's in such a way that every vertex belongs to $\left(d_{G}+d_{H}\right) /(k-1)$ graphs $K_{k}$ 's (obviously a covering of a subgraph $G$ of $K_{n}$ with $K_{k}$ 's is a set of $K_{k}$ 's containing all the edges of $G$ ).

The following proposition is immediate

\section{Proposition 1.1}

The degree $d_{G}$ of the regular leave $G$ of a regular packing of $K_{n}$ with $K_{k}$ 's satisfies the following equalities:

$$
\begin{aligned}
& n-1-d_{G} \text { an } 0(\bmod k-1) \\
& n\left(n-1-d_{G}\right)=0(\bmod k(k-1)) .
\end{aligned}
$$

The degree $d_{H}$ of the regular excess $H$ of a regular covering of $K_{n}$ with $K_{k}$ 's satisfies the following equalities:

$$
\begin{aligned}
& n-1+d_{H} \text { 표 } 0(\bmod k-1) \\
& n\left(n-1+d_{H}\right)=0(\bmod k(k-1))
\end{aligned}
$$

From that proposition, one can easily deduce lower bounds on $d_{G}$ and $d_{H}$.

Here we show that these bounds are always reached in the cases $k=3$ or 4 by constructing maximum regular packings and minimum regular coverings of $K_{n}$ with $K_{3}$ 's and $K_{4}$ 's for any $n$ (except for $n=8$ in case of a packing with $K_{4}$ 's). In most of the cases the covering constructed contains the packing. 
Let us remark that in some cases the optimum packings or coverings given in the literature $([5,13,14])$ are necessarily regular (by counting arguments). However as we want, if possible, to give a covering containing the packing, we give the construction again.

\section{Notation and basic lemmas}

- $K_{n}$ will denote the complete graph on $n$ vertices. Usually $V\left(K_{n}\right)$, the vertex set of $K_{n}$, will be $Z_{n}$, the group of integers modulo $n$.

- $\left[x_{1}, x_{2}, \ldots, x_{r}\right]$ will denote the complete graph on the vertices $x_{1}, x_{2}, \ldots, x_{r}$. In case $r=2$ it denotes an edge.

- $\left[x_{1}, x_{2}, \ldots, x_{r}\right](\bmod n)$ will denote $\left\{\left[x_{1}+i, x_{2}+i, \ldots, x_{r}+i\right]: i=0,1, \ldots, n-1\right\}$.

- $K_{n}-G$ denotes the partial graph obtained from $K_{n}$ by deleting the edges of $G$ from $K_{n}$.

- If $G$ and $H$ are graphs on the same set of vertices $V, G+H$ denotes the multigraph with vertex set $V$ containing the edges of $G$ plus the edges of $H$ (with eventually multiple edges).

- $K_{r_{1}, r_{2}}$ denotes the complete bipartite graph with vertex sets of cardinality $r_{1}$ and $r_{2}$.

- $K_{r_{1}, r_{2} . ., r_{h}}$ denotes the complete multipartite graph. If $r_{1}=r_{2}=\ldots=r_{h}=r$ then we denote $K_{r_{1}, r_{2}, ., r_{k}}$ by $K_{h \times r}$.

- Let $G$ be a graph on $k$ vertices. A parallel class of $G$ 's on $n=p k$ vertices is the vertex disjoint union of $p$ copies of $G$.

It is well known that the existence of a decomposition of $K_{h \times r}$ into $K_{h}$ 's is equivalent to the existence of $h-2$ orthogonal Latin squares of order $r$ (or also to what is called a $G D(h, 1, r ; h r)$ or $T(1, r)$ design). Classical results are the following.

Lemma 2.1 (see Hanani [8])

$K_{h \times r}$ can be decomposed into $K_{3}$ 's if and only if $\left\{\begin{array}{l}(h-1) r \equiv 0(\bmod 2) \\ h r^{2}(h-1)=0(\bmod 6) \\ h \geq 3\end{array}\right.$

Lemma 2.2 (see Brouwer, Hanani, Schrijver [4] )

$K_{h \times r}$ can be decomposed into $K_{4}^{\prime}, s$ if and only if

$$
\left\{\begin{array}{l}
(h-1) r \equiv 0(\bmod 3) \\
h r^{2}(h-1) \equiv 0(\bmod 12) \\
h \geq 4,(h, r) \neq(4,2) \text { or }(4,6)
\end{array}\right.
$$


Lemma 2.3 (see Bermond, Huang, Rosa, Sotteau [1])

If $K_{r, r, r}$ and $K_{r, r, r, r}$ can be decomposed into graphs $G$, then $K_{p r, p r, p r, q r}$ can be decomposed into $G$ for $p \neq 2,6,0 \leq q \leq p$ (and if $q=0$ for any $p$ ).

If $K_{r, r, r, r}$ and $K_{r, r, r, r, r}$ can be decomposed into graphs $G$, then $K_{p r, p r, p r, p r, g r}$ can be decomposed into $G$ for $p \neq 2,3,6,10$ and $0 \leq q \leq p$.

In [10] Huang, Mendelsohn and Rosa introduce a problem which can be formulated as that of the existence of a decomposition of $K_{n}$ into graphs all isomorphic to $K_{k}$ except one isomorphic to $K_{r}$. They give the necessary conditions.

The following results have been proved on this problem, which will be used in the proof of the main theorem.

Lemma 2.1 (Brouwer [5])

$K_{n}$ can be decomposed into one $K_{7}$ and $K_{1}$ 's if and only if $n \equiv 7$ or $10(\bmod 12)$, $n \neq 10,19$.

Lemma 2.5 (Bermond, Bond [3])

$K_{n}$ can be decomposed into one $K_{10}$ and $K_{4}$ 's if and only if $n=7$ or 10 (mod 12), $n \neq 7,19,22$.

In the section 5 we study a more particular case of this problem, since we want to have the additional property that there exists, in the decomposition, a parallel class of $K_{k}$ 's on $n-r$ vertices.

\section{Main results}

Theorem 3.1

If $n \geq 3$ there exist a maximum regular packing and a minimum regular covering of $K_{n}$ with $K_{3}{ }^{\prime} s$ where the graph $G$ and multigraph $H$ are as follows.
o) if $n \equiv 0 \quad(\bmod 6), \quad G$ is 1-regular and $H$ is 1-regular
i) if $n=1 \quad($ mod 8$), \quad G$ and $H$ are empty
ii) if $n \equiv 2 \quad(\bmod B), \quad G$ is 1-regular and $H$ is 5-regular
iii) if $n=3 \quad(\bmod B), \quad G$ and $H$ are empty
iv) if $n \equiv 4 \quad(\bmod 8), \quad G$ is 3-regular and $H$ is 3-regular
v) if $n \equiv 5 \quad(\bmod 6), \quad G$ is 4-regular and $H$ is 2-regular

Moreover there exists a minimum cosering containing a maximum packing in all савев except case o)

Proof

First, from Proposition 1.1, the given values of the degrees of $G$ and $H$ are lower bounds. Now we will prove that these bound are reached. 
Case o) $(n=6 t, t \geq 1)$ :

Since $K_{6 t+1}$ can be decomposed into $K_{3}$ 's, we get a packing of $K_{n}$ by deleting a vertex and all the $K_{3}$ 's containing it. The leave of the packing is a perfect matching.

It is known (see Mills [14] for a survey on the subject) that $K_{n}$ can be covered with $n / \beta[(n-1) / 2] \mid K_{3}$ 's. In this case the covering is regular and the excess $H$ is a perfect matching.

Obviously in this case it is not possible that the minimum covering contains the maximum packing since $G$ and $H$ are necessarily perfect matchings and the sum of two perfect matchings cannot be decomposed into $K_{3}$ 's.

Case i) $(n=6 t+1, t \geq 1)$ and case iii) $(n=6 t+3, t \geq 0)$ :

It is known that, in these cases, the graph $K_{n}$ can be decomposed into $K_{3}$ 's.

Case ii) $(n=6 t+2, t \geq 1)$ :

The packing is obtained exactly as in o), and the leave $G$ is a perfect matching. A regular covering of $G$ with each vertex in three $K_{3}$ 's is obtained by Corollary 4.2 applied to the 3-regular graph formed by the sum of $G$ and any 2-regular graph on the same set of vertices.

Case iv) $(n=6 t+4, t \geq 0)$ :

We distinguish two subcases:

i) $t=2 r, r \geq 0$

If $r=0$ then $n=4, G=K_{4}$ is itself 3 -regular. We can take $H=K_{4}$ since the multigraph formed by two identical $K_{1}$ 's can be decomposed into $K_{3}$ 's , $[0,1,2](\bmod 3)$.

If $r \geq 1, K_{12 r+1}$ is the edge disjoint union of $3 r+1$ vertex disjoint graphs $K_{4}$ 's and a $K_{(3 r+1) \times 4}$. By Lemma $2.1 K_{(3 r+1) \times 4}$ can be decomposed into $K_{3}$ 's. Therefore the leave $G$ consists of the $3 r+1$ vertex disjoint $K_{1}$ 's. We shall take for the excess $H$ the same graph. As the multigraph formed by two identical $K_{1}$ 's can be decomposed into $K_{3}$ 's, $G+H$ can also be decomposed into $K_{3}$ 's.

ii) $t=2 r+1, r \geq 0$

If $r=0$ then $n=10$. A maximum regular packing consists of the following $K_{3}$ 's:

$[0,3,8],[1,4,5],[2,0,6],[3,1,7],[4,2,8]$,

$[0,7,8],[1,8,0],[2,8,5],[3,5,6],[4,6,7]$.

The leave $G$ is the Petersen graph which is 3-regular. From Corollary 4.2 it can be covered with each vertex in three $K_{3}$ 's. So we have a covering of $K_{10}$ containing the maximum packing where the excess $H$ is a 3-regular multigraph.

If $r=1$ then $n=22$. The packing is given by the $K_{3}$ 's:

$$
[0,1,3],[0,4,10] \text { and }[0,5,13](\bmod 22) \text {. }
$$

The leave $G$ is 3 -regular and consists of the edges $[0,7]$ and $[0,11](\bmod 22)$. It is the union of a hamiltonian cycle and a perfect matching so, by Corollary 4.2 , it can be covered with each vertex in three $K_{3}$ 's. Thus we have a covering of $K_{22}$ containing the packing with an excess $H$ which is 3-regular.

If $r \geq 2, K_{12 r+10}$ can be decomposed into $r$ graphs $K_{12}$ 's on vertex sets $X_{i}, 1 \leq i \leq r$, one $K_{10}$, on vertex set $Y$ and a $K_{12, \ldots, 12,10}$ on vertex set $\bigcup X_{i} \bigcup^{Y}$. 
From above the $K_{10}$ can be decomposed into $K_{3}$ 's plus the 3-regular Petersen graph.

From case o) each $K_{12}$ on vertex set $X_{i}$ can be decomposed into $K_{3}$ 's plus a perfect matching $M_{i}$ for $1 \leq i \leq r$.

Now $K_{12, \ldots, 12,10}$ can be obtained from the multipartite graph $K_{(r+1) \times 12}$ by deleting two vertices in the same part. As from Lemma 2.1, $K_{(r+1) \times 12}$ can be decomposed into $K_{3}$ 's, the graph $K_{12, \ldots, 12,10}$ can be decomposed into $K_{3}$ 's plus two perfect matchings $M$ and $M^{\prime}$ on $\bigcup X_{i}$. Now we have a regular packing of $K_{12 r+10}$ where the leave $G$ is the 3-regular graph on $12 r+10$ vertices which consists of two parts: one is the Petersen graph on 10 vertices, the other one is on $12 r$ vertices: $\left\{\bigcup M_{i}+M+M^{\prime}\right\}$. According to Corollary 4.2 , both parts can be covered by $K_{3}$ 's in such a way that each vertex belongs to three $K_{3}$ 's.

Thus we have a covering of $K_{12 r+10}$ containing the packing with an excess $H$ which is 3-regular.

Case v) $(n=6 t+5, t \geq 0)$ :

If $t=0$ then $n=5$. We have $G=K_{5}$ and $H=C_{5}$, by covering $G$ with the $K_{3}$ 's $[0,1,2](\bmod 5)$.

If $t=1$ then $n=11$. The packing is given by the $K_{3}$ 's $[0,1,3](\bmod 11)$. The leave $G$ is the 4-regular formed by the edges $[0,4]$ and $[0,5]$ (mod 11). We obtain the covering by adding the $K_{3}$ 's $[0,1,5](\bmod 11)$. Note that the excess $H$ is a hamiltonian cycle.

If $t \geq 2, K_{6 t+5}$ is the edge disjoint union of $t$ vertex disjoint $K_{6}$ 's, on vertex sets $X_{i}$, $1 \leq i \leq t$, one $K_{5}$ on vertex set $Y$ and a multipartite graph $K_{6, \ldots, 6,5}$. According to Lemma 2.1, there exists a decomposition of $K_{(t+1) \times 6}$ into $K_{3}$ 's. By deleting one vertex we obtain a decomposition of $K_{6, \ldots, 6,5}$ into $K_{3}$ 's and a perfect matching $M$ on the vertex set $\cup X_{i}$.

Each $K_{6}$ on vertex set $X_{i}$ is the edge disjoint union of two vertex disjoint $K_{3}$ 's and a 3-regular graph which consists of a perfect matching $M_{i}$ and a cycle of length six $C_{6}^{i}$.

$K_{5}$ is itself a 4-regular graph on $Y$. So we have a maximum regular packing of $K_{6 t+5}$ with $K_{3}$ 's, where the leave $G$ is the 4-regular graph which consists of two parts: one is $\left\{\bigcup M_{i}+M+\cup C_{6}^{i}\right\}$, on vertex set $\bigcup X_{i}$, the other one is a $K_{5}$, on vertex set $Y$.

Both parts can be covered with each vertex in three $K_{3}$ 's, the $K_{5}$ as in case $n=5$ and the other one as shown in Corollary 4.3.

Thus we have a covering of $K_{0 t+5}$ containing the packing with an excess $H$ which is 2-regular.

\section{Remark 3.2}

The referee suggested to mention the following conjecture which seems very difficult.

\section{Conjecture}

For every given degree $d$, all d-regular graphs meeting the necessary conditions are leaves of a packing of $K_{n}$ with $K_{3}$ 's, with finitely many exceptions.

Note that the case of hamiltonian cycles was solved by E. Mendelsohn [11] and the general case of any 2-regular graph is a particular case of a result of Colbourn and Rosa [6] also obtained by Hilton and Rodgers [0]. 
Note that the above conjecture is in fact a special case of the following one of Nash-Williams [15].

Conjecture (Nash-Williams)

If $G$ is a graph of order $n, n \geq 15$, such that its number of edges is a multiple of 3 , and every vertex has an even degree at least $\frac{3 n}{4}$, then $G$ can be decomposed into $K_{3}$ 's.

\section{Remark 3.3}

K. Heinrich drew our attention to the following strong recent result of Rees [17] which can be restated as follows:

Theorem (Rees)

$K_{6 t}$ can be decomposed into $\alpha$ perfect matchings and $\beta$ parallel classes of $K_{3}$ 's if and only if $\alpha+2 \beta=6 t-1$ except for $\alpha=1$ and $t=1$ or $t=2$ (corresponding to the nonexistence of Nearly Kirkman triple systems of order 6 or 12).

Using this theorem we can give a shorter proof of the existence of maximum regular packings for the non immediate cases $n=6 t+u(u=4,5)$ in the following way. Let $V\left(K_{n}\right)=X \cup Y$ with $|X|=6 t,|Y|=u$. By the theorem above the $K_{b t}$ on $X$ can be decomposed into $2 u-1$ perfect matchings on $X$ and $3 t-u$ parallel classes of $K_{3}$ 's. Then $K_{n}$ can be decomposed into the $K_{u}$ on $Y, u-1$ perfect matchings on $X$ and $K_{3}$ 's formed by the parallel classes of $K_{3}$ 's on $X$ and the $3 t u$ triples obtained by joining any vertex of $Y$ to a perfect matching of $X$. Thus we have a regular packing of $K_{n}$ with $K_{3}$ 's for which the leave $G$ consists of the $K_{u}$ on $Y$ plus the $u-1$ perfect matchings on $X$ and is clearly a regular graph of degree $u-1$.

In fact we used only a corollary of the result of Rees. We needed only to decompose $K_{6 t}$ into perfect matchings and triangles (Note that such a decomposition solves the conjecture mentioned in Remark 3.2, when the graph is the union of $d$ perfect matchings). 
If $n \geq 4$, there exist a maximum regular packing and a minimum regular covering of $K_{n}$ with $K_{4}$ 's where the leave $G$ and excess $H$ are as follows.

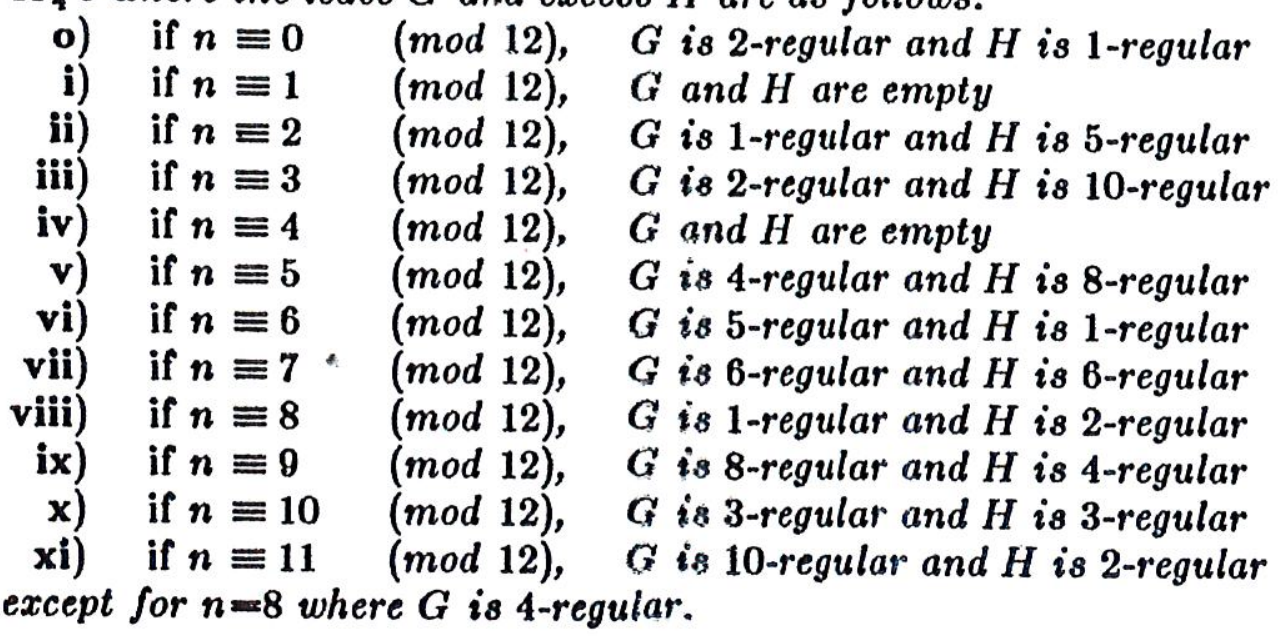

Moreover there exists a minimum regular covering which contains a maximum regular packing in all cases except o) and xi) and $n=18$ (where we don't know whether it exists).

\section{Proof:}

First, from Proposition 1.1, the given values of the degrees of $G$ and $H$ are lower bounds (except for $n=8$ ). Now we will prove that these bounds are reached.

case o) $(n=12 t, t \geq 1)$ :

We obtain a decomposition of $K_{12 t}$ into $K_{4}$ 's and a parallel class of $K_{3}$ 's by deleting one vertex and the edges containing it from a decomposition of $K_{12 t+1}$ into $K_{4}$ 's. Thus we have a maximum regular packing with a leave $G$ which is 2-regular.

A union of $K_{3}$ 's cannot be covered with $K_{4}$ 's with each vertex in only one $K_{4}$. In order to be able to find a minimum covering containing the minimum packing we would need to have a decomposition of $K_{12 t}$ into $K_{4}$ 's and a parallel class of cycles of length four. Such a decomposition can not exist for $n=12$. Maybe it does for bigger $n$, and we can state the following problem.

Problem : Does there exists a decomposition of $K_{12 t}$ into $K_{4}$ 's and a parallel class of $C_{4}$ 's for $t \geq 2$ ?

However it is known that there exists a maximum regular packing of $K_{12 t}$ with $K_{4}$ 's where the excess $H$ is a perfect matching. Indeed it has been proved in [12] that there exists a covering of $K_{12 t}$ with $12 t^{2}$ graphs $K_{4}$ 's. Since each vertex belongs to $4 t K_{4}$ 's, the excess $H$ is a perfect matching and the covering is regular.

case i) $(n=12 t+1, t \geq 1)$ and case iv) $(n=12 t+4, t \geq 0)$ :

It is known that, in these cases, the graph $K_{n}$ can be decomposed into $K_{4}$ 's.

case ii) $(n=12 t+2, t \geq 1)$ :

$K_{12 t+2}$ is the edge disjoint union of a perfect matching $M$ and a multipartite graph $K_{(6 t+1) \times 2}$. From Lemma $2.2, K_{(6 t+1) \times 2}$ can be decomposed into $K_{4}$ 's. Let us take 
$G=M=\{[2 i, 2 i+1], 0 \leq i \leq 6 t\}$. We have a covering of $G$ with each vertex in two $K_{4}$ 's by taking $\{[2 i, 2 i+1,2 i+2,2 i+3], 0 \leq i \leq 6 t\}$.

So we have a covering of $K_{12 t+2}$ containing the packing where the excess $H$ is a 5-regular multigraph.

case iii) $(n=12 t+3, t \geq 1)$ :

$K_{12 t+3}$ is the edge disjoint union of a parallel class of $K_{3}$ 's and a multipartite graph $K_{(4 t+1) \times 3}$. By Lemma 2.2, $K_{(4 t+1) \times 3}$ can be decomposed into $K_{4}$ 's. Let us take for $G$ the parallel class of $K_{3}$ 's which is a 2-regular graph. By Corollary 4.4 in section 4, we know that any 6-regular graph that can be decomposed into $K_{3}$ 's can be covered by $K_{4}$ 's with each vertex in four $K_{4}$ 's. We apply this result with the 6-regular graph formed by $G$ and any two other parallel classes of $K_{3}$ 's.

So we have a covering of $K_{12 t+3}$ containing the packing where the excess $H$ is a 10-regular multigraph.

case $v)(n=12 t+5, t \geq 0)$ :

First we prove the result for $n=5$ and 17 .

If $n=5$ then $G=K_{5}$ and $H$ is the 8-regular multigraph formed by two copies of $K_{5}$. Indeed, the graph $G+H$ can be decomposed into $K_{1}$ 's by taking $[0,1,2,4](\bmod 5)$.

If $n=17$, the packing is given by the following $K_{1}$ 's: $[0,1,4,6](\bmod 17)$. The leave $G$ is formed by the edges $[0,7]$ and $[0,8](\bmod 17)$. It is the union of two hamiltonian cycles and can be covered with each vertex in four $K_{4}$ 's by $[0,2,5,9](\bmod 17)$.

Now let $n=12 t+5$, with $t \geq 2$. By Lemma 2.4, $K_{12 t+7}$ can be decomposed into one $K_{7}$ and $K_{4}$ 's, if $t \neq 1$. We take such a decomposition and delete two vertices of the $K_{7}$. We obtain a decomposition of $K_{12 t+5}$ into $K_{5}$, two parallel classes of $K_{3}$ 's (on the $12 t$ other vertices) and $K_{4}$ 's. So the leave $G$ we obtain consists of two parts, one is a $K_{5}$ and the other one is a 4-regular graph on $12 t$ vertices, which is the union of two parallel classes of $K_{3}$ 's.

The $K_{5}$ can be covered as in the case $n=5$. By Corollary 4.4 in section 4, any 6-regular graph that can be decomposed into $K_{3}$ 's can be covered by $K_{4}$ 's, with each vertex in four $K_{4}$ 's. We apply this result to the 6-regular graph formed by the part of $G$ on $12 t$ vertices and any other parallel class of $K_{3}$ 's on the same set of vertices.

So we have a covering of $K_{12 t+5}$ containing the packing where the excess $H$ is a 8-regular multigraph.

case vi) $(n=12 t+6, t \geq 0)$ :

First we prove the result for $n=6$ and 18 .

If $n=6$ then $G=K_{6}$ is itself 5 -regular and $H$ is a perfect matching. The covering is given by the $K_{4}$ 's: $[0,1,2,3],[0,1,4,5],[2,3,4,5]$.

If $n=18$, then a regular packing can be obtained by taking $[0,1,3,8](\bmod 18)$. An optimal covering (which is regular in that case) is given in [12] by Mills. We note that this covering does not contain any maximum regular packing and we don't know if such a covering does exist.

Now let $n=12 t+6$, with $t \geq 2$. The graph $K_{12 t+6}$ is the edge disjoint union of a parallel class of $K_{6}$ and a multipartite graph $K_{(2 t+1) \times 6}$. By Lemma $2.2, K_{(2 t+1) \times 6}$ can be decomposed into $K_{4}$ 's. Therefore we obtain a leave $G$ which is a parallel class of $K_{6}$ 's. We cover each $K_{6}$ of $G$ as in the case $n=6$ and the excess $H$ is a perfect matching. 
case vii) $(n=12 t+7, t \geq 0)$ :

First we prove the result for the cases $n=7,19$.

If $n=7$ then $G=K_{7}$ is itself a 6-regular graph. We can take $H=K_{7}$ since the multigraph formed by two copies of the same $K_{7}$ can be decomposed into $K_{4}$ 's as follows: $[0,1,2,4](\bmod 7)$.

If $n=19$, a maximum packing is given by $[0,1,3,8](\bmod 19)$ and the leave is a 6-regular graph $G$ which is an edge disjoint union of $K_{3}$ 's : $[0,4,10](\bmod 10)$. So by Corollary $4.4, G$ can be covered by $K_{1}$ 's with each vertex in four $K_{4}$ 's.

Now let $n=12 t+7$, with $t \geq 2$. By Lemma 2.5, $K_{12 t+10}$ can be decomposed into one $K_{10}$ and $K_{4}$ 's, if $t \neq 1$. We take such a decomposition and delete three vertices of the $K_{10}$. We obtain a decomposition of $K_{12 t+7}$ into a $K_{7}$, three parallel classes of $K_{3}$ 's (on the other $12 t$ vertices) and $K_{1}$ 's. So the leave $G$ we obtain consists of the vertex disjoint union of a $K_{7}$ and a 6-regular graph on $12 t$ vertices, which can be decomposed into $K_{3}$ 's.

The $K_{7}$ can be covered as in the case $n=7$. By Corollary 4.4 in section 4 , the part of $G$ which is 6-regular on $12 t$ vertices can be covered by $K_{4}$ 's, with each vertex in four $K_{4}$ 's .

So we have a covering of $K_{12 t+7}$ containing the packing where the excess $H$ is a 6-regular multigraph.

case viii) $(n=12 t+8, t \geq 0)$ :

First we prove the result for the case $n=8$.

If $n=8$ then there exists no regular packing of $K_{8}$ with $K_{4}$ 's with a leave $G$ 1-regular. Indeed, if such a packing exists $K_{8}-G$ should be decomposed into four $K_{4}$ 's with each vertex in two $K_{1}$ 's which is easily seen to be impossible. A maximum regular packing of $K_{8}$ is obtained with a 4-regular leave $G$ by taking two disjoint $K_{4}$ 's.

A minimum regular covering of $K_{8}$ with a 2-regular excess $H$ is given by the following $K_{4}$ 's (found in [13]):

$[1,2,3,4],[1,2,5,6],[1,2,7,8],[3,4,5,6],[3,4,7,8],[5,6,7,8]$

This covering contains the maximum regular packing since it contains two vertex disjoint $K_{4}$ 's.

Now let $n=12 t+8$, with $t \geq 1$. The graph $K_{12 t+8}$ is the disjoint union of a perfect matching and a multipartite graph $K_{(6 t+4) \times 2}$. From Lemma 2.2, if $t \geq 1, K_{(6 t+4) \times 2}$ can be decomposed into $K_{4}$ 's. Let $G$ be the matching $\{[2 i, 2 i+1\}: i=0,1, \ldots, 6 t+3\}$. It can be covered with each vertex in one $K_{4}$ with the following $K_{4}$ 's : $\{[4 i, 4 i+1,4 i+2,4 i+3]: i=0,1, \ldots, 3 t+1\}$. Note that the excess $H$ is the vertex disjoint union of cycles of length 4 .

case ix) $(n=12 t+9, t \geq 0)$ :

It is well known that $K_{12 t+13}$ can be decomposed into $K_{4}$ 's. If we choose a $K_{4}$ and delete its four vertices, we get a decomposition of $K_{12 t+9}$ into four parallel classes of $K_{3}$ 's and $K_{4}$ 's. So the leave $G$ is the 8-regular graph formed by four parallel classes of $K_{3}$ 's.

From Corollary 4.5, we have a covering of $G$ with each vertex in four $K_{4}$ 's.

So we have a covering of $K_{12 t+9}$ containing the packing where the excess $H$ is a 4-regular multigraph. 
case $x)(n=12 t+10, t \geq 0):$

If $t=0$ then $n=10$. The packing consists of the following $K_{4}$ 's:

$$
[0,2,8,9],[1,3,8,5],[2,4,5,6],[3,0,6,7],[4,1,7,8]
$$

The leave $G$ is the Petersen graph which is a 3-regular graph.

In order to cover $G$ with each vertex in two $K_{4}$ 's, we add the following $K_{4}$ 's:

$$
[0,1,5,7],[1,2,6,8],[2,3,7,8],[3,4,8,5],[4,0,9,6]
$$

If $t=1$ then $n=22$. Let $V\left(K_{22}\right)=Z_{2} \times Z_{11}$. A maximum regular packing is given by the following $K_{4}$ 's:

$$
\begin{gathered}
\{((0, i),(0, i+1),(0, i+3),(1, i)],[(0, i+9),(1, i),(1, i+1),(1, i+5)], \\
[(0, i),(0, i+5),(1, i+6),(1, i+9)]: 0 \leq i \leq 10\} .
\end{gathered}
$$

The leave $G$ is the union of two vertex disjoint cycles of length 11 and a perfect matching joining them. It can be covered by the following $K_{4}$ 's :

where each vertex is in two $K_{4}$ 's.

$$
\{[(0, i),(0, i+4),(1, i+5),(1, i+7)]: 0 \leq i \leq 10\}
$$

If $t \geq 2$, the graph $K_{12 t+10}$ is the edge disjoint union of one $K_{10}, 3 t$ vertex disjoint $K_{4}$ 's and a multipartite graph $K_{4,4, \ldots 4,10}$.

As $t \geq 2$, by Theorem 5.1, which will be proved in section 5 , there exists a decomposition of the multipartite graph $K_{4,4, \ldots, 1,10}$ into $K_{4}$ 's since the number of 4-sets is equal to $3 t$. From the case $n=10$, the graph $K_{10}$ is the edge disjoint union of $K_{4}$ 's and a Petersen graph. So the leave $G$ is the vertex disjoint union of a Petersen graph and a parallel class of $K_{4}$ 's.

In order to obtain a minimum regular covering (with an excess $H$ 3-regular) containing the packing given above we add two parallel classes of $K_{4}$ 's on $12 t$ points and the $K_{4}$ 's we used to cover the Petersen graph in $K_{10}$.

case $\mathrm{xi})(n=12 t+11 t \geq 0)$ :

First we prove the result for $t=0,1,2$.

If $t=0$ then $G=K_{11}$ is itself 10-regular. A minimum covering is given by $[0,1,4,6](\bmod 11)$. Note that the excess $H$ is a hamiltonian cycle.

If $t=1$ then $n=23$. A maximum regular packing with $G 10$-regular is obtained by taking the following $K_{4}$ 's : $[0,1,7,21](\bmod 23)$. In order to cover $G$ with each vertex in four $K_{4}$ 's we add the following ones : $[0,1,11,10](\bmod 23)$. Note that the excess $H$ is again a hamiltonian cycle.

If $t=2$ then $n=35$. A maximum regular packing is given by : $[0,1,4,14]$ and $[0,7,9,15](\bmod 35)$. A covering containing it is obtained by adding the following $K_{4}$ 's : $[0,1,12,17](\bmod 35)$. Note that the excess $H$ is a hamiltonian cycle.

Now let $n=12 t+11$, with $t \geq 3$, the graph $K_{12 t+11}$ is the edge disjoint union of $t$ vertex disjoint $K_{12}$ 's, one $K_{11}$ and the multipartite graph $K_{12,12, \ldots, 12,11}$. From Lemma 2.2, the graph $K_{(t+1) \times 12}$ can be decomposed into $K_{4}$ 's for $t \geq 3$. Therefore $K_{12,12, \ldots, 12,11}$ can be decomposed into one parallel class of $K_{3}$ 's on $12 t$ vertices and $K_{1}$ 's. Each $K_{12}$ is the union of three vertex disjoint $K_{4}$ 's and an 8-regular graph. Let us take for leave $G$ the 10-regular graph which consists of two parts, one is formed on $12 t$ vertices by these 8-regular graphs plus the parallel class of $K_{3}$ 's, the other one is the $K_{11}$. Thus we have a maximum regular packing.

The existence of a minimum regular covering has been proved in [13] where it is proved that there exists a covering with $(t+1)(12 t+11) K_{4}$ 's with each vertex in $4 t+4$ 
graphs $K_{1}$ 's. Therefore the excess $H$ is 2-regular and the covering is regular minimum. Notice that in this last case we don't know whether there exists a minimum regular covering which contains a minimum regular packing.

\section{Remark 3.5}

The proof of the theorem suggests a lot of decomposition problems. The most general one would be a problem analogous to the conjecture of remark 3.2 , i.e. that all $d$-regular graphs meeting the necessary conditions are leaves (respectively excesses) of a regular packing (respectively covering) of $K_{n}$ with $K_{1}$ 's. First interesting cases are when the leaves or excesses are hamiltonian cycles or more generally regular graphs of degree two, which generalize Mendelsohn's [11] or Colbourn and Rosa's [6] result. For example we state the following conjectures.

\section{Conjectures}

$K_{12 t}$ minus a hamiltonian cycle

$K_{12 t+3}$ minus a hamiltonian cycle

$K_{12 t+8}$ plus a hamiltonian cycle

$K_{12 t+11}$ plus a hamiltonian cycle

can be decomposed into $K_{1}$ 's

One can also ask for a generalization of Rees' theorem.

\section{Conjecture}

$K_{12 t}$ can be decomposed into $\alpha$ parallel classes of $K_{3}$ 's and $\beta$ parallel classes of $K_{4}$ 's if and only if $2 \alpha+3 \beta=12 t-1$ (with a finite number of exceptions).

If we don't want parallel classes of $K_{4}$ 's we obtain the problem introduced by Huang, Mendelsohn and Rosa [10]. A related problem is considered in section 5.

Note that the problem of decomposing $K_{n}$ into $\alpha$ perfect matchings and $\beta$ parallel classes of $K_{4}$ 's for $n \equiv 0$ ( $\left.\bmod 4\right)$ is easy to solve (essentially because a parallel class of $K_{4}$ 's is the union of 3 perfect matchings).

\section{Some lemmas}

We state here some lemmas used in the proofs of the main theorem. The results are not necessarily best possible, and they are given in the form used in section 3 . 


\section{Lemma 4.1 :}

i) Let $G$ be an $r(r-1)$-regular graph on $n$ vertices that can be decomposed into $K_{r}$ 's. Then $G$ can be covered with $K_{r+1}$ 's, in such a way that each vertex belongs to $r+1$ graphs $K_{r+1}$ 's.

ii) Let $G$ be an $r(r-1)+1$-regular graph on $n$ vertices that can be decomposed into $K_{r}$ 's and a perfect matching. Then $G$ can be covered with $K_{r+1}$ 's, in such a way that each vertex belongs to $r+1$ graphs $K_{r+1}$ 's.

iii) Let $G$ be an $r(r-1)+2-$-regular graph on $n$ vertices that can be decomposed into $K_{r}$ 's and a 2-regular graph. Then $G$ can be covered with $K_{r+1}$ 's, in such a way that each vertex belongs to $r+1$ graphs $K_{r+1}$ 's.

Proof

First we remark that in case iii) the 2-regular graph is the vertex disjoint union of cycles, and one can give an orientation to the cycles so that every vertex is the origin of only one arc. Any vertex of $G$ is contained in $r$ graphs $K_{r}$ 's of the decomposition , so the total number of $K_{r}$ 's is $n$. Let us add one vertex of $G$ to each of the $K_{r}$ 's in order to transform it into a $K_{r+1}$, with each vertex of $G$ added exactly once. Obviously after that transformation we have $n$ graphs $K_{r+1}$ 's, with each vertex of $G$ belonging to $r+1$ of them. In order to do that and to have all the edges of $G$ covered we need a bijection $f$ between $V(G)$ and the set of the $n K_{r}$ 's, such that for any vertex $x$ of $G$ :

- in case i): $x$ does not belong to $f(x)$.

- in case ii): if $[x, y]$ is an edge of the matching, then $y \in f(x)$.

- in case iii): if $[x, y]$ is the arc having $x$ as origin in the 2-regular oriented partial graph of $G$, then $y \in f(x)$.

In order to show that such a bijection exists we define a bipartite graph, having as stable sets of vertices the $K_{r}$ 's and the vertices of $G$. We put an edge between a vertex of $G$ and a $K_{r}$ if the vertex can be added to the $K_{r}$. The result is a regular bipartite graph (of degree $n-r, r$ and $r$ respectively in cases i), ii) and iii)). A well-known corollary of the König-Hall theorem states that a regular bipartite graph admits a perfect matching. The matching defines the bijection we need.

We state now some corollaries of this lemma, which are used in section 3 , in these terms.

\section{Corollary 4.2 :}

Let $G$ be a 3-regular graph containing a perfect matching. Then $G$ can be covered by $K_{3}$ 's, in such a way that each vertex belongs to three $K_{3}$ 's.

\section{Corollary 4.3 :}

Let $G$ be a 4-regular graph" containing two disjoint perfect matchings. Then $G$ can be covered by $K_{3}$ 's, in such a way that each vertex belongs to three $K_{3}$ 's. 


\section{Corollary 4.4:}

Let $G$ be a 6-regular graph that can be decomposed into $K_{3}$ 's. Then $G$ can be covered by $K_{4}$ 's, in such a way that each vertex belongs to four $K_{4}$ 's.

\section{Corollary 4.5 :}

Let $G$ be an 8-regular graph that can be decomposed into $K_{3}$ 's and a 2-regular graph. Then $G$ can be covered by $K_{4}$ 's, in such a way that each vertex belongs to four $K_{4}$ 's.

\section{A multipartite graph decomposition problem}

We propose to study the following problem:

\section{Problem}

For what values of $h$ and $r$ can the multipartite graph $K_{4,4, \ldots, 4, r}$ on $4 h+r$ vertices be decomposed into $K_{4}$ 's $P$

If the multipartite graph $K_{4,4, \ldots, 4, r}$ on $4 h+r$ vertices can be decompused into $K_{4}$ 's then necessarily

$$
\left\{\begin{array}{l}
h \equiv 0(\bmod 3) \\
r \equiv 1(\bmod 3) \\
h \geq r / 2+1
\end{array}\right.
$$

We obtain the first two necessary conditions by divisibility arguments and the third one by counting the edges covered by the $K_{4}$ 's containing an element of the r-set.

We can remark that Lemma 2.2 proves that the above necessary conditions are sufficient for the case $r=4$ (this case can also be seen as a corollary of the well known theorem of Hanani [8], which says that $K_{n}$ can be decomposed into parallel classes of $K_{4}$ 's if and only if $\left.n \equiv 4(\bmod 12)\right)$.

Here we will prove that the above necessary conditions are sufficient for the case $r=10$.

We conjecture that the necessary conditions are sufficient in general, except eventually for a few values (for example it can be shown that $K_{4,4,4,1}$ cannot be decomposed into $K_{4}$ 's). 
Theorem 5.1

$K_{4,4, \ldots, 4,10}$ can be decomposed into $K_{4}$ 's if and only if $h$, the number of 4-vertex parts, satisfies

$$
\left\{\begin{array}{l}
h \equiv 0(\bmod 3) \\
h \geq 6
\end{array}\right.
$$

or equivalently

$K_{n}$ can be decomposed into one $K_{10}$, a parallel class of $K_{4}$ 's on the other $n-10$ vertices and $K_{4}$ 's if and only if $n \equiv 10(\bmod 12), n \neq 22$. or also,

$K_{n}$ can be decomposed into a parallel class of $K_{4}{ }^{\prime}, 10$ parallel classes of $K_{3}$ 's and $K_{4}$ 's if and only if $n \equiv 0(\bmod 12), n \neq 12$.

In what follows we will use the three alternative forms.

One can easily see, on the third formulation of the theorem, that it is a refinement of Lemma 2.5.

To prove the theorem we will need some composition lemmas, for which we will use the following remark:

\section{Remark 5.2}

$K_{t, t, t, t}$ can be decomposed into parallel classes of $K_{1}$ 's, for $t \neq 2,3,6,10$.

\section{Proof}

It is well known that $K_{t, t, t, t, t}$ can be decomposed into $K_{5}$ 's if and only if $t \neq 2,3,6,10$ (see [8]). Given such a decomposition we can delete the five vertices of a stable set of $K_{t, t, t, t, t}$. Each deleted vertex gives rise to a parallel class of $K_{4}$ 's in $K_{t, t, t, t}$.

We have the following composition lemmas.

\section{Lemma 5.3}

Let $t \geq u$, if $K_{12 t}$ and $K_{12 u}$ can be decomposed into one parallel class of $K_{1}$ 's, 10 parallel classes of $K_{3}$ 's and $K_{4}$ 's then so do $K_{48 t}$ and $K_{48 t+12 u}$.

\section{Proof}

The graph $K_{48 t+12 u}$ is the edge disjoint union of four $K_{12 t}$ 's, one $K_{12 u}$ and the multipartite graph $K_{12 t, 12 t, 12 t, 12 t, 12 u}$. The $K_{12 t}$ 's and the $K_{12 u}$ can be decomposed into 10 parallel classes of $K_{3}$, one parallel class of $K_{4}$ and $K_{4}$ 's by hypothesis. By Lemma 2.2, $K_{4 \times 12}$ and $K_{5 \times 12}$ can be decomposed into $K_{4}$ 's. Therefore, according to Lemma 2.3, $K_{12 t, 12 t, 12 t, 12 t, 12 u}$ can be decomposed into $K_{4}$ 's for $u \leq t$. Therefore $K_{48 t+36}$ can be decomposed into 10 parallel classes of $K_{3}$ 's , one parallel class of $K_{4}$ 's , and $K_{1}$ 's. 


\section{Lemma 5.4}

If $K_{12 t+9}$ can be decomposed into 10 parallel classes of $K_{3}$ 's and $K_{4}$ 's then $K_{48 t+36}$ can be decomposed into a parallel class of $K_{4}$ 's, 10 parallel classes of $K_{3}$ 's and $K_{4}^{\prime} s$.

\section{Proof}

$K_{48 t+36}$ is the disjoint union of four $K_{12 t+9}$ and the multipartite graph $K_{12 t+9,12 t+9,12 t+9,12 t+9}$. By hypothesis the $K_{12 t+9}$ can be decomposed into 10 parallel classes of $K_{3}$ 's and $K_{4}$ 's. As $12 t+9 \neq 2,3,6,10$, by applying Remark 5.2 , there exists a decomposition of $K_{12 t+9,12 t+9,12 t+9,12 t+9}$ into parallel classes of $K_{4}$ 's.

\section{Lemma 5.5}

If $K_{12 t+3}$ can be decomposed into 7 parallel classes of $K_{3}$ 's and $K_{4}$ 's then $K_{48 t+12}$ can be decomposed into a parallel class of $K_{4}$ 's, 10 parallel classes of $K_{3}$ 's and $K_{4}^{\prime} s^{\prime}$. Proof

Let $X=X_{1} \bigcup X_{2} \bigcup X_{3} \bigcup X_{4}$ with $\left|X_{i}\right|=12 t+4$. By Remark 5.2 the multipartite graph $K_{12 t+4,12 t+4,12 t+4,12 t+1}$ on vertex set $X$ can be decomposed into a parallel class of $K_{4}$ 's and $K_{1}$ 's as $12 t+4 \neq 2,3,6,10$. Choose a particular $K_{4},\left\{x_{1}, x_{2}, x_{3}, x_{4}\right\}$ with $x_{i} \in X_{i}$. Then the edges of the $K_{48 t+12}$ constructed on $X-\left\{x_{1}, x_{2}, x_{3}, x_{4}\right\}$ can be partitioned into the four $K_{12 t+3}$ on vertex set $X_{i}-x_{i}$, each decomposable into 7 parallel classes of $K_{3}$ 's and $K_{4}$ 's by hypothesis, the $K_{4}$ 's of $K_{12 t+4,12 t+4,12 t+4,12 t+4}$ not containing $x_{i}$ for any $i$ and the $K_{3}$ 's obtained from the $K_{4}$ 's containing one of the vertices $x_{i}$ when deleting it. The $K_{3}$ 's of this decomposition can be partitioned into 10 parallel classes of $K_{3}$ 's on $48 t+12$ vertices in the following way:

6 parallel classes, each of them being the union of parallel classes on each $X_{i}-x_{i}$, 4 parallel classes obtained for $i=1,2,3,4$ by taking, for each $i$, the parallel class still unused on $X_{i}-x_{i}$ and by adding the parallel class of $K_{3}$ 's on $\bigcup_{j \neq i}\left(X_{j}-x_{j}\right)$

obtained from the $K_{4}$ 's containing $x_{i}$ after deletion of $x_{i}$.

\section{Proof of Theorem 5.1.}

First the given conditions are necessary as we saw before. To prove that they are sufficient, by using Lemma 5.3, we only have to prove that $K_{n}$ can be decomposed into a parallel class of $K_{4}$ 's, 10 parallel classes of $K_{3}$ 's and $K_{4}$ 's, for $n=12 t$ with $t=2,3,4,5,6,7,9,11,13,17,21$ in order to finish the proof. From Lemma 5.4 and Lemma 2.5 we get the result with $t=7,11$. From Lemma 5.5 and Lemma 2.4 we get the result with $t=5,9,13,17,21$.

In what follows we will give the direct constructions for the remaining cases $(t=2,3,4,6)$.

The case $t=2$ :

For $n=24$, the decomposition of $K_{24}$ into a parallel class of $K_{4}$ 's and 10 parallel classes of $K_{3}$ 's is as follows. Let $V\left(K_{24}\right)=Z_{6} \times Z_{4}$; the vertices are labeled $(i, j)$ with $i=0,1, \ldots, 5$ and $j=0,1,2,3$. The decomposition is given below: 


$\begin{array}{lll}\text { Classes of } K_{3} \text { 's: } & & \\ {[(0, j),(1, j),(2, j)]} & {[(3, j),(4, j+1),(5, j)]} & \text { for } j=0,1,2,3 \\ {[(0, j),(1, j+1),(3, j)]} & {[(2, j),(4, j),(5, j+1)]} & \text { for } j=0,1,2,3 \\ {[(0, j),(1, j+2),(4, j)]} & {[(2, j),(3, j+2),(5, j)]} & \text { for } j=0,1,2,3 \\ {[(0, j),(1, j+3),(5, j)]} & {[(2, j),(3, j+3),(4, j+2)]} & \text { for } j=0,1,2,3 \\ {[(0, j),(2, j+1),(3, j+1)]} & {[(1, j),(4, j+1),(5, j+3)]} & \text { for } j=0,1,2,3 \\ {[(0, j),(2, j+2),(4, j+1)]} & {[(1, j),(3, j+1),(5, j+2)]} & \text { for } j=0,1,2,3 \\ {[(0, j),(2, j+3),(5, j+1)]} & {[(1, j),(3, j+2),(4, j)]} & \text { for } j=0,1,2,3 \\ {[(0, j),(3, j+2),(4, j+2)]} & {[(1, j),(2, j+1),(5, j)]} & \text { for } j=0,1,2,3 \\ {[(0, j),(3, j+3),(5, j+2)]} & {[(1, j),(2, j+2),(4, j+3)]} & \text { for } j=0,1,2,3 \\ {[(0, j),(4, j+3),(5, j+3)]} & {[(1, j),(2, j+3),(3, j)]} & \text { for } j=0,1,2,3 \\ \text { Class of } K_{4} \text { 's: } & & \\ {[(i, 0),(i, 1),(i, 2),(i, 3)]} & & \text { for } i=0, \ldots, 5\end{array}$

The case $t=3$ :

For $n=36$ the decomposition of $K_{36}$ into 10 parallel classes of $K_{3}$ 's and 5 parallel classes of $K_{4}$ 's is as follows. Let $V\left(K_{36}\right)=Z_{4} \times Z_{9}$; the vertices are labeled $(i, j)$ with $i=0,1,2,3$ and $j=0,1, \ldots, 8$. The decomposition uses the known decomposition of $K_{9}$ into 4 parallel classes of $K_{3}$ 's $([16])$. We form 8 parallel classes in the following way: for each $t=0,1,2,3$ we take 2 parallel classes on the 27 vertices $(i, j), i \neq t$ with two parallel classes of the $K_{9}$ constructed on the vertices $(t, j)$. The last two parallel classes are formed with the two unused classes on each $K_{9}$.

$\begin{array}{ll}\text { Classes of } K_{3} \text { 's: } & \\ {[(0, j),(1, j+7),(2, j+3)]} & \text { for } j=0, \ldots, 8 \\ {[(0, j),(1, j+8),(2, j+6)]} & \text { for } j=0, \ldots, 8 \\ {[(0, j),(1, j+4),(3, j+2)]} & \text { for } j=0, \ldots, 8 \\ {[(0, j),(1, j+6),(3, j+7)]} & \text { for } j=0, \ldots ., 8 \\ {[(0, j),(2, j+4),(3, j+1)]} & \text { for } j=0, \ldots, 8 \\ {[(0, j),(2, j+7),(3, j+6)]} & \text { for } j=0, \ldots, 8 \\ {[(1, j),(2, j+4),(3, j+6)]} & \text { for } j=0, \ldots, 8 \\ {[(1, j),(2, j+6),(3, j+4)]} & \text { for } j=0, \ldots, 8 \\ \text { a class from }(i, j) & \text { for } i=0,1,2,3 \\ \text { a class from }(i, j) & \text { for } i=0,1,2,3 \\ \text { Classes of } K_{4}, \mathrm{~s}: & \text { for } j=0, \ldots, 8 \\ {[(0, j),(1, j),(2, j),(3, j)]} & \text { for } j=0, \ldots, 8 \\ {[(0, j),(1, j+1),(2, j+2),(3, j+3)]} & \text { for } j=0, \ldots, 8 \\ {[(0, j),(1, j+2),(2, j+1),(3, j+5)]} & \text { for } j=0, \ldots, 8 \\ {[(0, j),(1, j+3),(2, j+5),(3, j+8)]} & \text { for } j=0, \ldots, 8 \\ {[(0, j),(1, j+5),(2, j+8),(3, j+4)]} & \end{array}$

and a class from $(3, j)$ and a class from $(3, j)$ and a class from $(2, j)$ and a class from $(2, j)$ and a class from $(1, j)$ and a class from $(1, j)$ and a class from $(0, j)$ and a class from $(0, j)$

The case $t=4$ :

For $n=48$ the decomposition of $K_{48}$ into 10 parallel classes of $K_{3}$ 's, a parallel class of $K_{4}$ 's and $K_{4}$ 's is as follows. Let $V\left(K_{48}\right)=Z_{48}$. 
Classes of $K_{3}$ 's:

$[i, i+16, i+32]$

$[i, i+1, i+5]$

$[i, i+1, i+5]$

$[i, i+1, i+5]$

$[i, i+2, i+13]$

$[i, i+2, i+13]$

$[i, i+2, i+13$

$[i, i+7, i+26]$

$[i, i+7, i+26]$

$[i, i+7, i+26]$ for $i=0, \ldots, 15$

for $i \equiv \mathbf{0}(\bmod 3)$

for $i \equiv 1(\bmod 3)$

for $i \equiv 2(\bmod 3)$

for $i \equiv 0(\bmod 3)$

for $i \equiv 1(\bmod 3)$

for $i \equiv 2(\bmod 3)$

for $i \equiv 0(\bmod 3)$

for $i \equiv 1(\bmod 3)$

for $i \equiv 2(\bmod 3)$
Class of $K_{1}$ 's:

$[i, i+12, i+24, i+36] \quad$ for $i=0, \ldots, 11$

$K_{4}$ 's:

$[i, i+3, i+20, i+30] \quad$ for $i=0, \ldots, 47$

$[i, i+6, i+14, i+30] \quad$ for $i=0, \ldots, 47$

The case $t=6$ :

For $v=72$ the decomposition of $K_{72}$ into 10 parallel classes of $K_{3}$ 's, a parallel class of $K_{4}$ 's and $K_{4}$ 's is as follows. Let $V\left(K_{72}\right)=Z_{72}$.

Classes of $K_{3}$ 's:

$[i, i+24, i+48]$

$[i, i+1, i+5]$

$[i, i+1, i+5]$

$[i, i+1, i+5]$

$[i, i+2, i+10]$

$[i, i+2, i+10]$

$[i, i+2, i+10]$

$[i, i+7, i+20]$

$[i, i+7, i+20]$

$[i, i+7, i+20]$

for $i=0, \ldots, 23$

for $i \equiv 0(\bmod 3)$

Class of $K_{4}$ 's:

$[i, i+18, i+36, i+54] \quad$ for $i=0, \ldots, 17$

for $i \equiv 1(\bmod 3)$

for $i \equiv 2(\bmod 3)$

$K_{1}$ 's:

for $i=0(\bmod 3)$

for $i \equiv 1(\bmod 3)$

$[i, i+3, i+12, i+41]$

for $i=0, \ldots, 71$

for $i \equiv 2(\bmod 3)$

$[i, i+11, i+26, i+51]$

for $i=0, \ldots, 71$

$[i, i+14, i+30, i+49]$

for $i=0, \ldots, 71$

for $i \equiv 0(\bmod 3)$

$[i, i+6, i+33, i+50]$

for $i=0, \ldots, 71$

for $i \equiv 1(\bmod 3)$

for $i \equiv 2(\bmod 3)$

\section{Acknowledgement}

This research was partially supported by P.R.C. Math. Info.

\section{References}

1. J-C. Bermond, C. Huang, A. Rosa, and D. Sotteau, Decomposition of complete graphs into isomorphic subgraphs with five vertices, Ars Combinat. 10 pp. 211-

2. J-C. Bermond, J. Bond, and J-F. Sacle, Large hypergraphs of diameter one, in Graph Theory and Combinatorics, Proc. Coll. Cambridge, 1989, pp. 19-28 (1984).

3. J-C. Bermond and J. Bond, Combinatorial designs and hypergraphs of diameter one, in Proc. First China-USA Conf. on Graph Theory, Jinan,June 1986, (1987).

4. A.E. Brouwer, H. Hanani, and A. Schrijver, Group divisible designs with block size four, Discrete Math. 20 pp. 1-10 (1977).

5. A.E. Brouwer, Optimal packings of $K_{1}$ 's into a $K_{n}$, Journal of Comb. Th., ser. A 26 pp. 278-207 (1979). 
6. C.J. Colbourn and A. Rosa, Quadratic Leaves of Maximal Partial Triple Systems, Graphs and Combinatorics 2, pp. 317-337 (1986).

7. J. Doyen and A. Rosa, An updated bibliography and survey of Steiner systems, pp. 317-349 in Topics on Steiner systems, C.C. Lindner and A. Rosa ed., Annals

8. H. Hanani, Balanced incomplete block designs and related designs, Discrete Math. 11 pp. 255-369 (1975). 9. A.J.W. Hilton and C.A. Rodger, Triangulating nearly complete graphs of odd
order, in preparation, (1986).

10. C. Huang, E. Mendelsohn, and A. Rosa, On partially resolvable $t$-partitions, pp. 169-183 in Theory and Practice of Combinatorics, A. Rosa, G. Sabidussi and J. Turgeon ed., Annals of Discrete Math., 12, (1982).

11. E. Mendelsohn, On (Near)-Hamiltonian Triple Systems and related onefactorizations of complete graphs, Techrion Report MT-655, (1985).

12. W.H. Mills, On the covering of pairs by quadruples. I, Journal of Comb. Th., ser. $A 13$ pp. 55-78 (1872).

13. W.H. Mills, On the covering of pairs by quadruples. II, Journal of Comb. Th., ser. $A 15$ pp. 138-166 (1873).

14. W.H. Mills, Covering designs I: Coverings by a small number of subsets, Ars Combinat. 8 pp. 198-315 (1979).

15. C.St.J.A. Nash-Williams, An unsolved problem concerning decomposition of graphs into triangles, Technical report, University of Waterloo, (1969).

16. D. K. Ray-Chaudhuri and R. M. Wilson, Solution of Kirkman's schoolgirl problem, pp. 187-204 in Proc. of Symp. in Pure Math., vol 18 Combinatorics, Amer. Math. Soc. Providence (1971).

17. R. Rees, Uniformly resolvable pairwise balanced designs with blocksizes two and three, Preprint, Queen's University, Kingston, (1886).

18. R.M. Wilson, An existence theory for pairwise balanced designs, III: Proof of the existence conjectures, Journal of Comb. Th., ser. A 18 pp. 71-79 (1975). 